\title{
A Wireless Network Communication Capacity Control Technology Based on Fuzzy Wavelet Neural Network
}

\author{
Bing Zheng and Dawei Yun $(1)$ \\ Department of Information Engineering, Hainan Vocational University of Science and Technology, Haikou 571126, China \\ Correspondence should be addressed to Dawei Yun; yundw@hvust.edu.cn
}

Received 11 March 2021; Revised 24 April 2021; Accepted 10 May 2021; Published 26 July 2021

Academic Editor: Yong Deng

Copyright (C) 2021 Bing Zheng and Dawei Yun. This is an open access article distributed under the Creative Commons Attribution License, which permits unrestricted use, distribution, and reproduction in any medium, provided the original work is properly cited.

\begin{abstract}
The communication capacity control of the computer wireless network is the basis for realizing the efficient communication of massive data. In order to study the communication capacity control technology of the computer wireless network, improve the control effect of a large amount of data communication, and calculate the capacity of the wireless network in real time, this paper uses the fuzzy wavelet neural network to predict the wireless network channel. After the interference-free channel is obtained, the load balancing strategy of the ant colony optimization algorithm is used to filter the channel, and the channel allocation sequence with the most balanced load distribution is obtained, and a priority selection list is generated. After discretizing the channels in the largest discretization selection list, the channel sequence is allocated to the pair of nodes with communication requests according to the greedy coloring algorithm, so as to realize the communication capacity control of the computer wireless network. The test results show that the technology can guarantee good communication performance in both static and dynamic networks and can effectively complete network communication of massive data, and the communication capacity control effect is good.
\end{abstract}

\section{Introduction}

Wireless network communication capacity refers to the maximum amount of information that can be transmitted in a unit time, which is the embodiment of the advantages and disadvantages of wireless network communication ability, and it is greatly related to communication lines, work efficiency, and information passing time. At present, the Internet has covered people's daily life. With the continuous integration of wireless communication technology and computer network technology, computer network technology has also developed rapidly. It has developed from simple data transmission in the past to multimedia data transmission such as voice, image, and video [1]. With the rapid development of computer technology, network technology, and communication technology, wireless network communication performs the functions of traditional network communication, such as file transmission, peripheral sharing, Web browsing, e-mail, and database access. Its main characteristics are high data transmission rate, relatively low price, and fast network construction speed [2]. Because the wireless communication link has the characteristics of high burst error rate, signal attenuation and background noise, interference conflict, and so on and because of the distributed random access characteristics of DCF, it will be greatly affected by network congestion, resulting in communication bottlenecks and other problems [3]. Because the communication line is the main reason that affects the wireless network communication, the communication line is also called the channel, and the capacity of the channel determines the capacity of the network communication [4]. Channel capacity is a parameter of the channel, which can reflect the maximum amount of information that can be transmitted by the channel. At present, the single-channel protocol is the main protocol in the practical application of wireless network, which simplifies the implementation of the protocol, reduces the difficulty of mutual discovery between nodes, and makes the networking more rapid and convenient [5]. However, when the amount of data is very large, many nodes will apply for communication on this channel at the same time, 
resulting in increased communication competition. Therefore, it is studied in this paper, and the communication capacity control technology of computer wireless network is proposed, which is used to solve the problem of communication capacity control and ensure the efficient transmission of data communication [6].

The key contributions of this work are as follows:

(1) This paper uses fuzzy wavelet neural network to predict wireless network channels

(2) This paper uses the load balancing strategy of the ant colony optimization algorithm to filter the channels, obtain the channel allocation sequence with the most balanced load distribution, and generate a priority selection list

(3) The channel sequence is allocated to the pair of nodes with communication requests according to the greedy coloring algorithm to realize the communication capacity control of the computer wireless network

The rest of this paper is organized as follows: Section 2 discusses communication capacity control technology of computer wireless network, followed by the experimental test which is discussed in Section 3. Section 4 concludes the paper with summary and future research directions.

\section{Communication Capacity Control Technology of Computer Wireless Network}

In this paper, a multichannel MAC protocol is used, which can make many nodes in wireless sensor network and use multiple channels for parallel communication, so as to greatly improve the utilization of network bandwidth resources, reduce communication competition, and improve the network communication capacity [7].

The communication capacity control of computer wireless network is divided into three steps: channel prediction, screening, and allocation. In the communication process of computer wireless network, the prediction model of fuzzy wavelet neural network is used to get the channel without interference in the next stage [8]. Then, the load balancing strategy based on ant colony algorithm is applied to filter the channels, and the channel sequences without interference and with low load are recorded in the priority choice lists (PCL). Then, the maximum discretization algorithm is applied to select the channel from the PCL for channel discretization [9]. After the maximum discretization of the channel is completed, the channel sequence is assigned to the node pair with communication request to realize the communication capacity control of computer wireless network [10].

\subsection{Channel Prediction Based on Fuzzy Wavelet Neural} Network. Fuzzy wavelet neural network is composed of fuzzy neural network and wavelet network. The first half realizes a TSK fuzzy model, and the second half is wavelet network.
The outputs of the two network models are the outputs of the model [11]. The network structure is shown in Figure 1.

It can be seen from Figure 1 that the neural network has a three-layer structure including an input layer, a calculation layer, and an output layer. There is a close connection between each level. In the structure of fuzzy wavelet neural network, the number of nodes in fuzzy rules is the same as the number of input signals [12]. In the second layer, each node has its corresponding language rules [13]. The membership value of each input signal to the network belongs to a part of the fuzzy system set. The membership function is expressed by Gauss function as

$$
\mu_{j}\left(x_{i}\right)=\exp \left[-\frac{\left(x_{i}-\xi_{i j}\right)^{2}}{\tau_{i j}^{2}}\right], \quad i=1,2, \cdots, k, j=1,2, \cdots, m,
$$

in which the input signal variables of the fuzzy wavelet neural network, the number of nodes of the second layer network, and the center and width of the membership function of the $j$ th term of the $i$ th input are represented by $x_{1}, x_{2}, \cdots, x_{k}$ $; n ; \xi_{i j}$; and $\tau_{i j}$, respectively [14].

The output of the third layer of the fuzzy network can be expressed as follows:

$$
\mu_{\theta}(x)=\prod_{j} \mu_{j}\left(x_{i}\right) \theta=1,2, \cdots, n, \quad j=1,2, \cdots, m
$$

in which the output of the fuzzy network is $\mu_{\theta}(x)$, which is also the input signal of the final network. Therefore, the common output of the wavelet network and fuzzy network can be expressed as

$$
u=\frac{\sum_{\theta=1}^{n} \mu_{\theta}(x) y_{\theta}}{\sum_{\theta=1}^{n} \mu_{\theta}(x)}
$$

in which the output of the upper wavelet network and the final output of the network are $\mu_{\theta}(x) ; y_{1}, y_{2}, \cdots, y_{n}$; and $U$, respectively [15].

When using the fuzzy wavelet neural network to predict a channel, the spectrum state $\beta$ of binary sequence with length $T$ in the channel is decomposed into vector $\beta_{t-\tau+1}^{t}=\left[\beta_{t}\right.$, $\left.\beta_{t-1}, \cdots, \beta_{t-\tau+2}, \beta_{t-\tau+1}\right]$ with length $\tau$ as an input vector of the fuzzy wavelet network, the output is $Y^{\circ}$, and its equation is

$$
\begin{gathered}
\beta_{t}=\left[\begin{array}{ccccc}
\beta_{t} & \cdots & \beta_{t} & \cdots & \beta_{T-1} \\
\beta_{t-1} & \vdots & \beta_{t-1} & \vdots & \beta_{T-2} \\
\vdots & \vdots & \vdots & \vdots & \vdots \\
\beta_{2} & \vdots & \beta_{t-\tau+2} & \vdots & \beta_{T-\tau+1} \\
\beta_{1} & \cdots & \beta_{t-\tau+1} & \cdots & \beta_{T-t}
\end{array}\right], \\
Y^{\circ}=\left[\beta_{\tau+1}, \beta_{t+1}, \cdots, \beta_{T}\right] .
\end{gathered}
$$

Each column of $\beta_{t}$ is sent to the input layer neuron as the input sequence of the fuzzy wavelet neural network. Because 


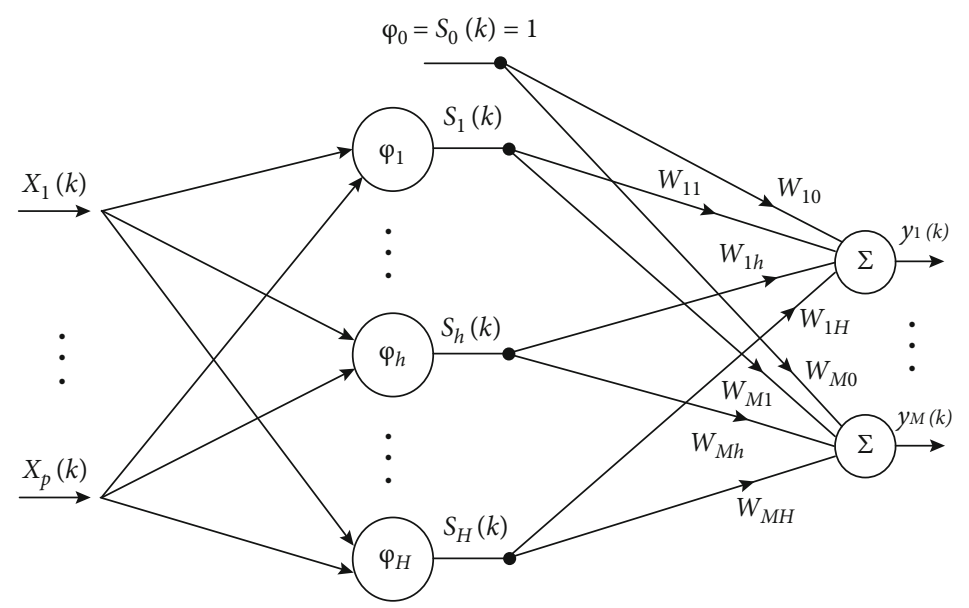

Figure 1: Structure of fuzzy wavelet neural network.

the determination of the fuzzy rules of the sample set affects the network structure and the initial parameters of the network, it can reduce the complexity and improve the generalization performance [16]. Therefore, we first extract the rules from the sample set and use the fuzzy $C$-means clustering algorithm to complete the rule extraction; that is, for $N$ samples $\left\{\left(x_{j}, y_{j}\right)\right\}, j=1,2, \cdots N$, the number of clusters $c$ is set; then, the problem is transformed into an optimization problem that meets the constraint conditions [17].

$$
\min _{\mu_{k l}, y_{j}} J=\sum_{j=1}^{N} \sum_{k=1}^{c}\left(\mu_{k j}\right)^{m} d_{k j}^{2} .
$$

The constraint conditions are as follows:

$$
\left\{\begin{array}{l}
\sum_{k=1}^{c} \mu_{k j}=1, \quad 1 \leq j \leq N \\
\mu_{k j} \geq 0, \quad 1 \leq j \leq N, 1 \leq k \leq c
\end{array}\right.
$$

in which the fuzzy index of clustering is expressed by $m$, and $m>1$; the membership degree of sample $x_{j}$ belonging to cluster $k$ is expressed by $\mu_{k j}$; the Euclidean distance and diagonal matrix between the sample point and cluster center are $d_{k j}^{2}=A_{j}(x)=\left(x_{i}-v_{k}\right)^{T}\left(F_{k}\right)^{-1}\left(x_{j}-v_{k}\right)$ and $F_{k}$, respectively [18].

Let the partition matrix and center matrix of clustering be $U=\left[\mu_{k j}\right], K=1,2, \cdots, C, J=1,2, \cdots, N$, and $V=\left[v_{1}, v_{2}, \cdots\right.$, $\left.v_{c}\right]$, and the number of cycles and the ending error be $l=1$ and $\varepsilon>0$, respectively. The process of fuzzy clustering is as follows:

(1) Calculate the fuzzy clustering model:

$$
v_{k}^{(l)}=\frac{\sum_{j=1}^{N}\left(\mu_{k i}^{(l-1)}\right)^{m} Z_{j}}{\sum_{j=1}^{N}\left(\mu_{k i}^{(l-1)}\right)^{m}}, \quad k=1,2, \cdots, c,
$$

in which $Z_{j}$ is sample set

(2) Calculate the covariance matrix:

$$
F_{k}=\frac{\sum_{j=1}^{N}\left(\mu_{k i}^{(l-1)}\right)^{m}\left(Z_{j}-v_{k}^{(l)}\right)\left(Z_{j}-v_{k}^{(l)}\right)^{T}}{\sum_{j=1}^{N}\left(\mu_{k i}^{(l-1)}\right)^{m}}
$$

(3) Calculate the distance to the cluster:

$d_{k j}^{2}=\left(Z_{j}-v_{k}^{(l)}\right)^{T} D_{k}\left(Z_{j}-v_{k}^{(l)}\right), \quad 1 \leq I \leq n, 1 \leq k \leq c$

(4) Update partition matrix:

$$
v_{k, j}^{(l)}=\frac{1}{\sum_{i=1}^{c}\left(D_{k, j}^{2}\left(z_{j}, v_{k}\right) / D_{i, j}^{2}\left(z_{j}, v_{i}\right)\right)^{1 /(m-1)}}
$$

(5) Using orthogonal least square method, according to the principle of maximum error change rate, $M_{s}$ important clusters are found and saved [19]. $M=M_{s} ; \quad U^{(l)}=\left[u_{i}\right], i=1, \cdots, M_{s} ; \quad$ and $\quad U \quad$ is regularized 
(6) If $\left\|U^{(l)}-U^{(l-1)}\right\|<\varepsilon$ ends, otherwise $l=l+1$, return (1) to continue running

For the generated fuzzy rule, if $x_{1}$ is $A_{1}^{j}$ and $x_{n}$ is $A_{n}^{j}$, then $i \widehat{y}_{j}=\widehat{Y}_{\mathrm{WNN}}$, where $R_{j}$ is the $j$ th fuzzy rule and $\widehat{y}_{j}$ is the output corresponding to the $j$ th fuzzy rule $R_{j}$. $A_{i}^{j}$ Gaussian membership function $\mu_{i j}$ is

$$
\mu_{i j}\left(\beta_{i}\right)=\exp \left(-\frac{\left(\beta_{i}-\xi_{i j}\right)^{2}}{\tau_{i j}^{2}}\right)
$$

In this case, the output rule $\mu_{j}$ can be expressed as

$$
\mu_{J}=\prod_{i=1}^{n} \exp \left(-\left(\frac{\left(\beta_{i}-\xi_{j, i}\right)}{\tau_{j, i}^{2}}\right)^{2}\right)
$$
follows:

The output $\widehat{Y}_{\text {WNN }}$ of the wavelet network is calculated as

$$
\widehat{Y}_{\mathrm{WNN}_{j}}=\sum_{a=1}^{M} \sum_{b=0}^{a} \omega_{a, b, j} \cdot \varphi_{a, b}\left(\sum_{i=1}^{n} C_{a, b}^{i} \cdot X_{i}\right)
$$

in which the scale factor and displacement factor are $a$ and $b$, respectively.

The prediction output of the whole network is $\widehat{Y}$ :

$$
\widehat{Y}=\frac{\sum_{j=1}^{M}\left(\mu_{j} \cdot \widehat{Y}_{\mathrm{WNN}_{j}}\right)}{\sum_{j=1}^{M} \mu_{j}}
$$

$C_{a, b}^{i}$ and $\omega_{a, b}$ use the least square difference of gradient descent.

$$
E=\frac{1}{2 i} \sum_{i=1}^{n}(\widehat{Y}-y(i))^{2}
$$

in which $E$ is the error and $y$ is the expected output.

The gradient descent method is used to adjust the parameters $\xi, \tau, C$, and weight $\omega$ in equations (10) and (14). The first two parameters depend on the fuzzy membership function, the last two parameters come from the wavelet network model, and $\alpha$ is the learning rate [20].

$$
\begin{gathered}
\tau(q+1)=\tau(q)-\left.\alpha \cdot \frac{\partial E}{\partial \tau}\right|_{q}=\tau(q)-\left.\alpha \cdot \frac{1}{i} \cdot\left(\widehat{Y}-Y_{i}\right) \cdot \frac{\partial \widehat{Y}}{\partial \tau}\right|_{q} \\
\xi(q+1)=\xi(q)-\left.\alpha \cdot \frac{\partial E}{\partial \xi}\right|_{q}=\xi(q)-\left.\alpha \cdot \frac{1}{i} \cdot\left(\widehat{Y}-Y_{i}\right) \cdot \frac{\partial \widehat{Y}}{\partial \xi}\right|_{q} \\
\omega(q+1)=\omega(q)-\left.\alpha \cdot(\widehat{Y}-y(i)) \cdot \frac{1}{i} \cdot \frac{1}{a} \cdot z_{j} \cdot \frac{\partial \widehat{Y}_{\mathrm{WNN}}}{\partial \omega}\right|_{q} \\
c(q+1)=c(q)-\left.\alpha \cdot(\widehat{Y}-y(i)) \cdot \frac{1}{i} \cdot \frac{1}{a} \cdot z_{j} \cdot \frac{\partial \widehat{Y}_{\mathrm{WNN}}}{\partial c}\right|_{q} .
\end{gathered}
$$

If node $j$ is connected to rule node $l$, then

$\frac{\partial \mu_{l}\left(\beta_{j}\right)}{\partial \xi_{j i}}=\mu_{l}\left(\beta_{j}\right) \frac{2\left(\beta_{j}-\xi_{j i}\right)}{\tau_{j i}^{2}}, \frac{\partial \mu_{l}\left(\beta_{j}\right)}{\partial \tau_{j i}}=\mu_{l}\left(\beta_{j}\right) \frac{2\left(\beta_{j}-\xi_{j i}\right)^{2}}{\tau_{j i}^{2}}$.

2.2. Load Balancing Based on Ant Colony Algorithm. Load balancing problem for wireless sensor network channel allocation is to make the data traffic balanced allocation to each channel. Load balancing is very important for the performance of communication capacity and average delay of wireless sensor networks [21]. The reason is that if the network load cannot be evenly distributed, the channel with large load will lead to the backlog of data services, while the channel with small load will produce the phenomenon that the channel is idle after the transmission of data services, thus affecting the communication capacity of wireless sensor networks [22]. In this paper, low overhead MAC protocol and ant colony optimization algorithm are used to integrate and exchange information between data links and networks, establish certain connections, work cooperatively, remove redundant operations, and allocate network resources more effectively [23].

In the algorithm, pheromone updating is divided into iterative optimal updating and local pheromone updating. The search path selection methods of searching solution space include two kinds: according to the prior law and according to the probability [24]. The purpose of this algorithm is as follows: at a certain time, when the data traffic set in the network comes, the ant colony algorithm is used to find a channel allocation sequence which makes the load most balanced distribution. Whether the channel load distribution is balanced or not can be expressed by the standard deviation of the channel load distribution [25].

$$
B=\sqrt{\frac{1}{n} \sum_{j=1}^{n}\left(x_{i}-q\right)^{2}}, \quad q=\frac{1}{n} \sum_{j=1}^{n} x_{j}
$$

in which $j$ is the total number of available channels, $x_{j}$ is the load ratio of channel $j, q$ is the average load ratio of channel, 
and $B$ is the standard deviation of the load ratio of channel. The smaller the value of $B$ is, the smaller the fluctuation of channel load is and the more balanced the load distribution is. The algorithm first updates the channel load ratio for the channel with low load [26]. With the increase of the overall load, the update mechanism gradually releases the restriction of the load ratio update, allowing more channels with relatively high load to join the algorithm scheduling; when the system load drops, the scheduling of individual channels with relatively high load will be closed in the global update [27], so as to achieve the purpose of overall load balancing.

(1) Iterate and update equation

$$
\begin{aligned}
\tau(r, s) & =(1-\rho) \times \tau(r, s)+\rho \Delta \tau(r, s), \\
\Delta \tau(r, s) & =\left\{\begin{array}{l}
K \sigma \tau(r, s)^{-1}, \quad(r, s) \in \mathrm{IOP}, \\
0, \quad \text { otherwise, }
\end{array}\right.
\end{aligned}
$$

in which $i(r, s)$ is the pheromone content of the channel $r s$, that is, the ratio of the load of the channel $r s$ to the total load; $\Delta i(r, s)$ is the increment of the ratio of the load on the channel to the total load; $\rho$ is the volatile factor of the iterative optimal update load ratio; and $(1-\rho)$ is the residual factor of the new load ratio. By changing the values of $\rho$ and $K$, the growth rate of channel load is adjusted [28]. $\sigma \tau(r, s)$ is the standard deviation of the optimal channel sequence load generated in each iteration, and the channel sequence with no interference and low load is recorded in the priority choice lists (PCL); IOP is the iterative optimal path $[29,30]$.

(2) Local update equation

$$
\tau(r, s)=(1-\vartheta) \tau(r, s)+\vartheta \tau(0),
$$

in which $\vartheta$ is the volatility coefficient of the local update load ratio, $\tau(0)$ is the initial value of the load ratio, and the introduction of $\vartheta$ can reduce the load ratio on the selected channel, which is conducive to the discovery of new paths [31].

(3) Path transfer rule equation

$$
S= \begin{cases}\arg \max \left\{[\tau(r, u)]^{\alpha} \cdot[\varphi(r, u)]^{\beta}\right\}, & q \leq q_{0}, \\ P_{i j}^{k}, & q>q_{0},\end{cases}
$$

in which $q_{0}\left(o<q_{0}<1\right)$ is a parameter of the algorithm, and $q_{0}$ is a random number between 0 and $1 . \alpha$ is the heuristic factor, which is the adjustment parameter of load ratio; $\beta$ is the expected heuristic factor, which is the adjustment parameter of visibility. $\varphi(r, u)$ is heuristic information. When $q>q_{0}$, the channel is searched according to the probability; when $q \leq q_{0}$, the channel is selected according to the prior law [32].

(4) Calculation equation of transition probability

$$
P_{i j}^{k}(t)= \begin{cases}\frac{\tau_{i j}^{\alpha}(t) * \varphi_{i j}^{\beta}(t)}{\sum_{\text {scallowed }_{k}} \tau_{\text {is }}^{\alpha}(t) * \varphi_{i j}^{\beta}(t)}, & j=\text { allpwed }_{k}, \\ 0, & \text { otherwise, }\end{cases}
$$

in which $P_{i j}^{k}(t)$ is the probability that the $k$ th ant selects channel $i j$ at time $t$, and allpwed $_{k}$ represents the set of nodes connected with the edge of node $i$, that is, the set of nodes that can be selected by the $k$ th ant at node $i$. If the node is in the set, its probability is calculated according to the concentration of load ratio; otherwise, the probability is 0 . The transfer probability equation indicates that the $k$ th ant of an iteration is calculated according to the proportion of the channel load to the total load. If the load of the channel accounts for the greater proportion of the total load, the probability of transfer is also greater [33].

The algorithm description of multichannel load balancing scheduling algorithm based on ant colony algorithm is as follows:

Step 1. Parameters are set to initialize the load ratio of the channel.

Step 2. The ants in the ant colony choose a channel according to the selection probability.

Step 3. If the load ratio of each channel after scheduling is less than the set threshold.

Step 4. The data service is allocated to the channel.

Step 5. The channel load ratio is updated according to the channel searched by ants in ant colony.

Step 6. If there is a channel after scheduling, the load ratio is greater than the set threshold.

Step 7. Return to Step 2, and reselect a channel.

The maximum discretization is used to process the channel in the PCL list. The equation is as follows:

$$
G(i)=[i+O H, i+1 H, i+k H], \quad i=1,2,3,4, k=3,
$$

in which $k$ is the maximum value satisfying the inequality $i$ $+k H \leq 16$. Each group includes at least $n$ channels, and the equation is as follows:

$$
n=\text { floor }\left(\frac{c}{H}\right) .
$$

In order to ensure the maximum discretization and the maximum separation of each group of channels, the channel expression equation of group $j$ is as follows:

$$
\operatorname{Group}(j)=[G(j) ; G(j+n) ; G(j+t n)] .
$$

Channel maximum discretization is completed. When 
TABLE 1: Details of wireless network node parameters.

\begin{tabular}{lcc}
\hline Parameter & The values & Meaning \\
\hline Chan & Channe/wirelessChanne & The channel type is a wireless channel \\
Prop & Propagation/FreeSpace & The transport model is free space \\
Mac & Mac/Mmac & Protocol used \\
ifqlen & 50 & Size of the interface queue \\
$X$ & 500 & Topological range length \\
$Y$ & 500 & Width of topology range \\
Stop & 100 & Set model time \\
nn & 200 & Number of wireless nodes \\
$N$ & 10 & The total number of channels \\
\hline
\end{tabular}

there is a node pair to transmit data, the channel is selected from the channel list after the maximum discretization according to the greedy coloring algorithm to assign the channel to the node pair, so as to control the communication capacity of the computer wireless network.

\section{Experimental Test}

In order to test the communication capacity control effect of the wireless network, NS2 simulation platform is used to complete the simulation experiment. During the experiment, the details of wireless network node parameters are shown in Table 1.

The performance of the multichannel MAC protocol has a great impact on the result of communication capacity control. Throughput average packet delay and average handshake time are used to measure the performance of the multichannel MAC protocol. One of the main goals of multichannel MAC protocol design is to increase the throughput of the network. The parameter can directly reflect the performance of the protocol. Packet delay refers to the time from the sending node to the receiving node, which is composed of queue delay, sending delay, and backoff delay. The average packet delay is an important index to measure the quality of a MAC protocol. The calculation equations are as follows:

Cumulative throughput

$$
=\frac{\text { Group size } \times \text { number of packets sent successfully }}{\text { Simulation time }},
$$

Average packet delay

$$
=\frac{\sum \text { Packet delay of successfully received packets }}{\text { Number of packets received successfully }} \text {. }
$$

The transmission performance of the proposed technology in static network and dynamic network is tested, and the performance of the multichannel MAC protocol and typical single transceiver multichannel protocol (MMAC protocol) is compared under different packet arrival rates. Comparison results of the two protocols in static network and dynamic network are shown in Table 2. Packet average delay results in static and dynamic networks are shown in Table 3.
TABLE 2: Comparison results of the two protocols in static network

\begin{tabular}{|c|c|c|c|}
\hline $\begin{array}{l}\text { Network } \\
\text { type }\end{array}$ & $\begin{array}{l}\text { Arrival } \\
\text { rate } \\
(\mathrm{Kbps})\end{array}$ & $\begin{array}{c}\text { This paper presents a } \\
\text { multichannel MAC } \\
\text { protocol }\end{array}$ & $\begin{array}{l}\text { Single transceiver } \\
\text { multichannel } \\
\text { MMAC protocol }\end{array}$ \\
\hline \multirow{11}{*}{$\begin{array}{l}\text { Static } \\
\text { network }\end{array}$} & 0 & 0 & 0 \\
\hline & 20 & 0.30 & 0.30 \\
\hline & 40 & 0.38 & 0.38 \\
\hline & 60 & 0.79 & 0.61 \\
\hline & 80 & 0.82 & 1.22 \\
\hline & 100 & 0.98 & 1.62 \\
\hline & 120 & 1.04 & 1.71 \\
\hline & 140 & 1.18 & 1.78 \\
\hline & 160 & 1.21 & 1.82 \\
\hline & 180 & 1.2 & 1.78 \\
\hline & 200 & 1.21 & 1.84 \\
\hline \multirow{11}{*}{$\begin{array}{l}\text { Dynamic } \\
\text { network }\end{array}$} & 0 & 0 & 0 \\
\hline & 20 & 0.28 & 0.30 \\
\hline & 40 & 0.31 & 0.41 \\
\hline & 60 & 0.42 & 0.68 \\
\hline & 80 & 0.79 & 1.12 \\
\hline & 100 & 0.88 & 1.29 \\
\hline & 120 & 1.22 & 1.71 \\
\hline & 140 & 1.22 & 1.54 \\
\hline & 160 & 1.21 & 1.61 \\
\hline & 180 & 1.2 & 1.77 \\
\hline & 200 & 1.21 & 1.78 \\
\hline
\end{tabular}
and dynamic network (bit).

According to the test results in Table 2, in the static network and dynamic network, when the packet arrival rate is lower than $40 \mathrm{Kbps}$, the throughput difference between the multichannel MAC protocol and the typical single transceiver multichannel protocol (MMAC protocol) is small; this phenomenon is because the network load is small, the multichannel can meet the transmission needs of the network, and there will be no more serious data conflict; when the packet arrival rate is lower than $40 \mathrm{Kbps}$, the throughput difference between the multichannel MAC protocol and the typical single transceiver multichannel protocol (MMAC protocol) is small; when the packet rate is higher than $60 \mathrm{Kbps}$, the 
TABle 3: Packet average delay results in static and dynamic networks (sec).

\begin{tabular}{|c|c|c|c|}
\hline $\begin{array}{l}\text { Network } \\
\text { type }\end{array}$ & $\begin{array}{l}\text { Arrival } \\
\text { rate } \\
(\mathrm{Kbps})\end{array}$ & $\begin{array}{c}\text { This paper presents a } \\
\text { multichannel MAC } \\
\text { protocol }\end{array}$ & $\begin{array}{l}\text { Single transceiver } \\
\text { multichannel } \\
\text { MMAC protocol }\end{array}$ \\
\hline \multirow{11}{*}{$\begin{array}{l}\text { Static } \\
\text { network }\end{array}$} & 0 & 0.01 & 0.01 \\
\hline & 20 & 0.02 & 0.02 \\
\hline & 40 & 0.03 & 0.03 \\
\hline & 60 & 0.03 & 0.03 \\
\hline & 80 & 0.03 & 0.03 \\
\hline & 100 & 0.04 & 0.08 \\
\hline & 120 & 0.05 & 0.12 \\
\hline & 140 & 0.05 & 0.18 \\
\hline & 160 & 0.07 & 0.27 \\
\hline & 180 & 0.12 & 0.28 \\
\hline & 200 & 0.18 & 0.33 \\
\hline \multirow{11}{*}{$\begin{array}{l}\text { Dynamic } \\
\text { network }\end{array}$} & 0 & 0 & 0 \\
\hline & 20 & 0.04 & 0.05 \\
\hline & 40 & 0.06 & 0.09 \\
\hline & 60 & 0.08 & 0.13 \\
\hline & 80 & 0.10 & 0.17 \\
\hline & 100 & 0.12 & 0.27 \\
\hline & 120 & 0.27 & 0.34 \\
\hline & 140 & 0.31 & 0.39 \\
\hline & 160 & 0.32 & 0.41 \\
\hline & 180 & 0.36 & 0.55 \\
\hline & 200 & 0.43 & 0.61 \\
\hline
\end{tabular}

throughput of the multichannel MAC protocol is significantly higher than that of the typical single transceiver multichannel protocol (MMAC protocol), and with the gradual increase of the packet arrival rate, the difference between the throughput of the two protocols becomes larger and larger. This is because the multichannel MAC protocol is based on the maximum discrete channel allocation, which can effectively avoid the mutual interference between adjacent channels and alternate channels and greatly improve the utilization of wireless network channel resources, so it can effectively increase the throughput of wireless network communication. Packet average delay results in static and dynamic networks are shown in Table 3.

According to the test results in Table 3 , in a static network, when the packet arrival rate is lower than $80 \mathrm{Kbps}$, the average delay of the multichannel MAC protocol and the typical single transceiver multichannel protocol (MMAC protocol) is equal; with the increase of the packet arrival rate, the load of the wireless network also increases; at this time, the average delay of the multichannel MAC protocol begins to be lower than the typical single transceiver multichannel protocol (MMAC protocol), and the average delay of the network is reduced greatly. In the dynamic network, the average delay of the two protocols increases slowly when the packet arrival rate is $20 \mathrm{Kbps}$, but the average delay of the multichannel MAC protocol is lower than that of the typical single

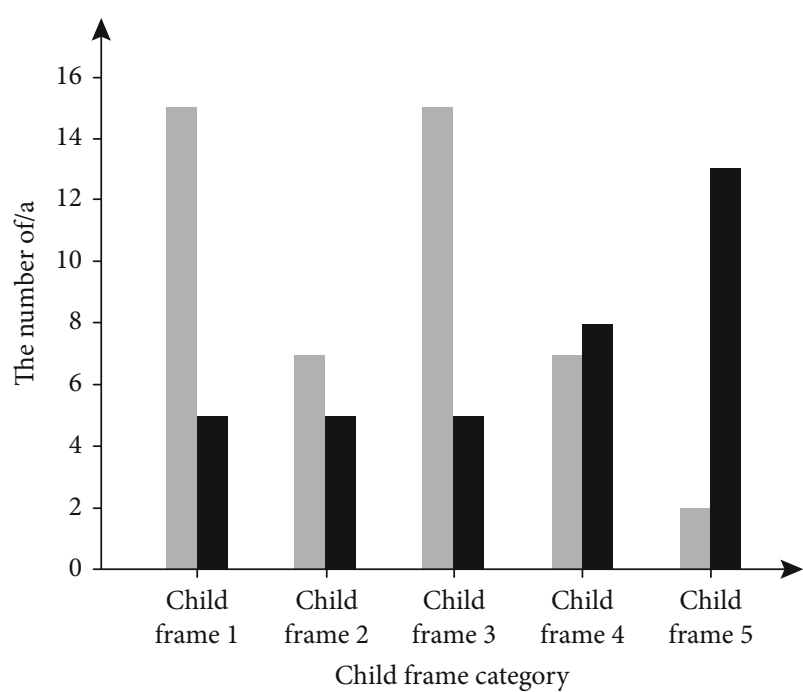

Use the number

Unused quantity

FIGURE 2: Wireless network communication capacity before control.

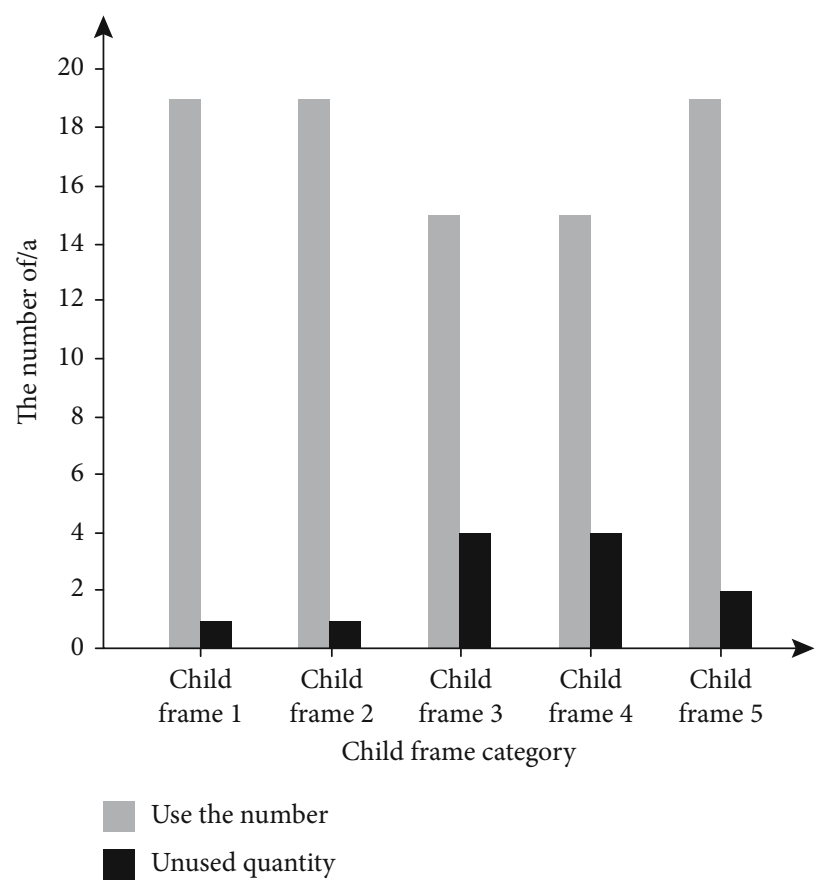

FIGURE 3: Wireless network communication capacity after control.

transceiver multichannel protocol (MMAC protocol). It shows that the transmission performance of the proposed technology is better.

In order to test the communication capacity control effect of the proposed method, the wireless network communication capacity before and after the control is counted. Wireless network communication capacity before control is shown in Figure 2. Wireless network communication capacity after control is shown in Figure 3.

According to the test results of Figures 3 and 4, before the control, there is a large amount of spare capacity in the 


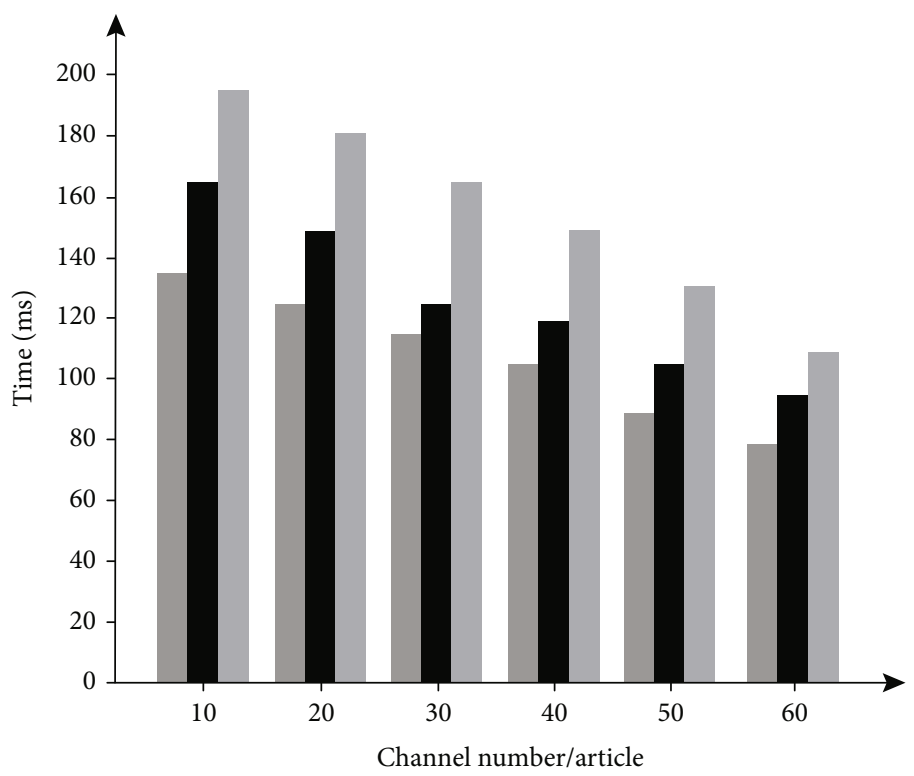

$2 \mathrm{gb}$ of data

$5 \mathrm{gb}$ of data

The data of $10 \mathrm{gb}$

FIgURE 4: Data transmission time before control.

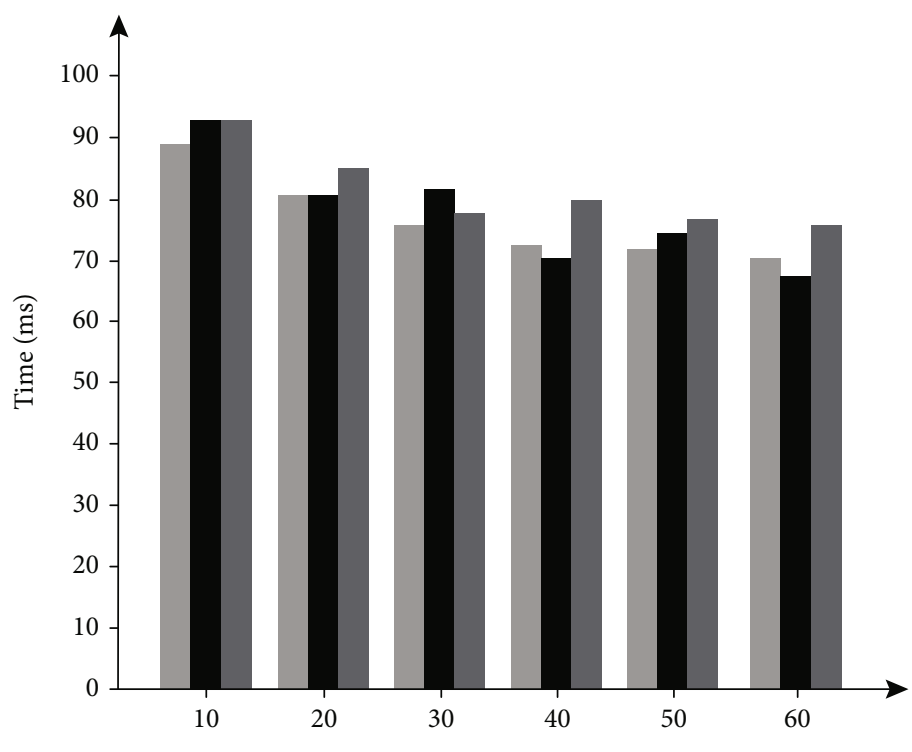

Channel number/article

$2 \mathrm{gb}$ of data

$5 \mathrm{gb}$ of data

The data of $10 \mathrm{gb}$

Figure 5: Data transmission time after control.

network, which does not make full use of the channel to complete the effective allocation of capacity; after using the technology in this paper, the use and allocation of spare capacity can be completed by using the channel reasonably. It shows that this technology has good control effect on the communi- cation capacity of computer wireless network and can make full use of the channel to achieve capacity allocation.

In the case of different numbers of channels, the time required for data transmission of different sizes before and after control is tested. Data transmission time before control 
is shown in Figure 4. Data transmission time after control is shown in Figure 5.

According to the test results of Figures 4 and 5, when the data volume is $2 \mathrm{~GB}, 5 \mathrm{~GB}$, and $10 \mathrm{~GB}$, the larger the data volume is before the communication capacity control, the more time-consuming the transmission is, because the larger the data volume is, many nodes will send communication applications to the channel at the same time, resulting in communication competition, and there will be interference between nodes, resulting in the increase of transmission timeconsuming; after the communication capacity control, it can realize multi-channel parallel communication, and improve the utilization of network broadband and transmission efficiency.

\section{Conclusion}

The communication capacity control of computer wireless network is the main means to meet the current communication needs of computer wireless network, so this paper studies the communication capacity control technology of wireless network based on fuzzy wavelet neural network and ant colony algorithm. In order to ensure good network connectivity as the premise, according to the channel load to carry out channel allocation, the reasonable use of the channel can be realized, to reduce the cost of nodes, so as to ensure the effective control of communication transmission capacity. The test results show that the technology is effective for the communication capacity control of computer wireless network, and the network communication performance is good. In the future research, how to calculate the capacity of wireless network communication in real time has become a main research direction. This link should be added to the research of this article.

\section{Data Availability}

The datasets used and/or analyzed during the current study are available from the corresponding author on reasonable request.

\section{Conflicts of Interest}

The authors declare that they have no conflict of interest.

\section{Acknowledgments}

This study was funded by Beichuang teaching assistant FundUniversity industry research innovation fund of Science and Technology Development Center, Ministry of Education (Grant No. 2018A05031).

\section{References}

[1] S. Shan, X. Duan, Y. Zhang, and T. T. Zhang, "Research on collaborative governance of smart government based on Blockchain technology: an evolutionary approach," Discrete Dynamics in Nature and Societ, vol. 21, no. 4, pp. 1-23, 2021.

[2] X. Hao, J. Liu, N. Yao, and L. Xie, "Research of network capacity and transmission energy consumption in WSNs based on game theory," Dianzi Yu Xinxi Xuebao/Journal of Electronics and Information Technology, vol. 40, no. 7, pp. 1715-1722, 2018.

[3] J. Cui and F. Zhang, "Design and development of GPS navigation and positioning system based on AutoCAD platform," Computer-Aided Design and Applications, vol. 18, no. S3, pp. 46-57, 2020.

[4] S. Sayed and A. Massoud, "General classification and comprehensive performance assessment of multi-objective DC voltage control in multi-terminal HVDC networks," IEEE Access, vol. 9, pp. 34454-34474, 2021.

[5] O. Kennedy, E. Chukwu, O. Shobayo, E. Noma-Osaghae, I. Okokpujie, and M. Odusami, "Comparative analysis of the performance of various active queue management techniques to varying wireless network conditions," International Journal of Electrical and Computer Engineering, vol. 9, no. 1, pp. 359$368,2019$.

[6] L. Jin, Z. Zhang, N. Wang, Z. Liu, and Y. Fu, "Intensity distribution of partially coherent array finite airy beams propagating in atmospheric turbulence," International Journal of Optics, vol. 2021, Article ID 6649144, 11 pages, 2021.

[7] H. Li, H. Ishidaira, K. SOUMA, and J. Magome, “Assessment of the flood control capacity and cost efficiency of sponge city construction in Mianyang City, China," Journal of Japan Society of Civil Engineers Ser G (Environmental Research), vol. 76, no. 5, pp. 46-57, 2020.

[8] F. R. Z. Hasibuan and I. Indayani, "The effect of application of SAK-ETAP, internal control system, and capacity of cooperatives on the development of business in cooperatives in Banda Aceh City," Journal of Accounting Research Organization and Economic, vol. 2, no. 3, pp. 262-270, 2020.

[9] E. B. Aleksandrova, A. A. Shtyrkina, and A. V. Yarmak, "Isogeny-based cryptographic access control," Automatic Control and Computer Sciences, vol. 54, no. 8, pp. 803-812, 2020.

[10] M. Yoshida, T. Kan, K. Kasai, T. Hirooka, K. Iwatsuki, and M. Nakazawa, "10 channel WDM $80 \mathrm{Gbit} / \mathrm{s} / \mathrm{ch}, 256$ QAM bi-directional coherent transmission for a high capacity nextgeneration mobile fronthaul," Journal of Lightwave Technology, vol. 39, no. 5, pp. 1289-1295, 2021.

[11] F. P. L. Sanico, "Organizational capacity assessment as input to policy direction in the University of Eastern Philippines, Catarman Northern Samar," International Journal of ResearchGRANTHAALAYAH, vol. 7, no. 3, pp. 120-129, 2020.

[12] C. Xia, Y. Zhang, and Y. Liu, "Path planning and energy flow control of wireless power transfer for sensor nodes in wireless sensor networks," Turkish Journal of Electrical Engineering and Computer Sciences, vol. 26, no. 5, pp. 2618-2632, 2018.

[13] Z. P. Liu, "Research on risk control of relay protection under Hainan Power Grid with large capacity generators," Yunnan Electric Power Technology, vol. 47, no. 12, pp. 63-66, 2019.

[14] M. Sivaram and V. Porkodi, "FCCP-NS: a fair congestion control protocol with N-sinks in wireless sensor networks," International Journal of Advanced Trends in Computer Science and Engineering, vol. 8, no. 12, pp. 43-51, 2019.

[15] G. F. Liu, X. S. Han, Y. Tian, and H. K. Li, "Research on prevention and control technology of oxide scale of boiler in 350 MW supercritical unit," Reneng Dongli Gongcheng/Journal of Engineering for Thermal Energy and Power, vol. 33, no. 8, pp. 144-148, 2018.

[16] C. M. Meng and H. J. Liang, "Research on existing problems in maintenance and management of mobile communication 
network," Digital technology and application, vol. 37, no. 1, pp. 36-38, 2020.

[17] T. A. Podshibyakina, "Political discourse in online communities as a communication technology of network control," Opcion, vol. 35, no. 23, pp. 1301-1314, 2020.

[18] B. G. Ibrahimov, M. Q. Hasanov, R. T. Humbatov, and R. F. Ibrahimov, "Research on the performance of intelligent communication networks," IFAC-PapersOnLine, vol. 51, no. 30, pp. 768-770, 2020.

[19] Y. Qiu, H. Zhang, K. Long, and M. Guizani, "Subchannel assignment and power allocation for time-varying fog radio access network with NOMA," IEEE Transactions on Wireless Communications, vol. 3, no. 8, pp. 1-1, 2021.

[20] R. Zhou, B. Li, M. Yang, and Z. J. Yan, "Simulation research on multi-user media access control technology for the next generation WLAN," Computer Simulation, vol. 36, no. 8, pp. 288292, 2019.

[21] Z. Niu, B. Zhang, J. Wang et al., "The research on $220 \mathrm{GHz}$ multicarrier high-speed communication system," China Communications, vol. 17, no. 3, pp. 131-139, 2020.

[22] B. Zhang, Z. Niu, J. Wang et al., "Four-hundred gigahertz broadband multi-branch waveguide coupler," IET Microwaves, Antennas \& Propagation, vol. 14, no. 11, pp. 11751179, 2020.

[23] B. Zhang, D. Ji, D. Fang, S. Liang, Y. Fan, and X. Chen, "A novel $220-\mathrm{GHz} \mathrm{GaN}$ diode on-chip tripler with high driven power," IEEE Electron Device Letters, vol. 40, no. 5, pp. 780783, 2019.

[24] A. Li, D. Spano, J. Krivochiza et al., "A tutorial on interference exploitation via symbol-level precoding: overview, state-ofthe-art and future directions," IEEE Communications Surveys and Tutorials, vol. 22, no. 2, pp. 796-839, 2020.

[25] J. Zhang and G. Qu, "Physical unclonable function-based key sharing via machine learning for IoT security," IEEE Transactions on Industrial Electronics, vol. 67, no. 8, pp. 7025-7033, 2019.

[26] J. Zhang and C. Shen, "Set-based obfuscation for strong PUFs against machine learning attacks," IEEE Transactions on Circuits and Systems. I: Regular Papers, vol. 68, no. 1, pp. 288300, 2021.

[27] J. Shi, Y. Lu, and J. Zhang, "Approximation attacks on strong PUFs," IEEE Transactions on Computer-Aided Design of Integrated Circuits and Systems, vol. 39, no. 10, pp. 2138-2151, 2020.

[28] P. Wang and Y. Liu, "SEMA: secure and efficient message authentication protocol for VANETs," IEEE Systems Journal, vol. 15, no. 1, pp. 846-855, 2021.

[29] B. Li, Y. Liu, A. Zhang, W. Wang, and S. Wan, "A survey on blocking technology of entity resolution," Journal of Computer Science and Technology, vol. 4, no. 35, pp. 769-793, 2020.

[30] Q. Jiang, F. Shao, W. Gao, Z. Chen, G. Jiang, and Y. Ho, "Unified no-reference quality assessment of singly and multiply distorted stereoscopic images," IEEE Transactions on Image Processing, vol. 28, no. 4, pp. 1866-1881, 2019.
[31] Y. Sun, C. Xu, G. Li et al., "Intelligent human computer interaction based on non redundant EMG signal," Alexandria Engineering Journal, vol. 59, no. 3, pp. 1149-1157, 2020.

[32] D. Jiang, G. Li, Y. Sun, J. Hu, J. Yun, and Y. Liu, "Manipulator grabbing position detection with information fusion of color image and depth image using deep learning," Journal of Ambient Intelligence and Humanized Computing, vol. 4, 2021.

[33] F. Xiao, G. Li, D. Jiang et al., "An effective and unified method to derive the inverse kinematics formulas of general six-DOF manipulator with simple geometry," Mechanism and Machine Theory, vol. 159, article 104265, 2021. 